University of Nebraska - Lincoln

DigitalCommons@University of Nebraska - Lincoln

$11-15-1994$

\title{
Interface alloying and magnetic properties of Fe/Rh multilayers
}

\author{
K. Hanisch \\ Laboratorium fir Angewandte Physik, Universitat Duisburg, Germany \\ W. Keune \\ Laboratorium fir Angewandte Physik, Universitat Duisburg, Germany \\ R.A. Brand \\ Laboratorium fir Angewandte Physik, Universitat Duisburg, Germany \\ Christian Binek \\ University of Nebraska-Lincoln, cbinek@unl.edu \\ Wolfman Kleemann \\ Laboratorium fir Angewandte Physik, Universitat Duisburg, Germany, wolfgang.kleemann@uni-due.de
}

Follow this and additional works at: https://digitalcommons.unl.edu/physicsbinek

Part of the Physics Commons

Hanisch, K.; Keune, W.; Brand, R.A.; Binek, Christian; and Kleemann, Wolfman, "Interface alloying and magnetic properties of Fe/Rh multilayers" (1994). Christian Binek Publications. 4.

https://digitalcommons.unl.edu/physicsbinek/4

This Article is brought to you for free and open access by the Research Papers in Physics and Astronomy at DigitalCommons@University of Nebraska - Lincoln. It has been accepted for inclusion in Christian Binek Publications by an authorized administrator of DigitalCommons@University of Nebraska - Lincoln. 


\section{Interface alloying and magnetic properties of $\mathrm{Fe} / \mathrm{Rh}$ multilayers}

K. Hanisch, W. Keune, R. A. Brand, C. Binek, and W. Kleemann

Laboratorium für Angewandte Physik, Universität Duisburg, D-47048 Duisburg, Germany

$\operatorname{Rh}(20 \AA) f^{57} \mathrm{Fe}\left(t_{\mathrm{Fe}}\right)$ multilayers with Fe thicknesses $t_{\mathrm{Fe}}$ of $2,5,10$, and $15 \AA$ prepared by alternate evaporation in UHV have been investigated by x-ray diffraction (XRD), Mössbauer spectroscopy, and SQUID magnetometry. First- and second-order superstructure Bragg peaks (but no higher-order peaks) in small-angle XRD patterns suggest some compositional modulation. Mössbauer spectra taken at $4.2 \mathrm{~K}$ are characterized by a distribution $P\left(B_{\mathrm{h} f}\right)$ of hyperfine fields $B_{\mathrm{hf}}$. Peaks observed in the $P\left(B_{\mathrm{hf}}\right)$ curves near 17 and $35 \mathrm{~T}$ are assigned to an fcc-RhFe interface alloy $(\sim 7-24$ at. \% $\mathrm{Fe})$ with spin-glasslike properties and to a disordered ferromagnetic bcc-FeRh alloy ( $\sim 96$ at. $\% \mathrm{Fe}$ ), respectively. The magnetic transition temperature of the fcc alloy was found to be 23 and $45 \mathrm{~K}$ for $t_{\mathrm{Fe}}=2$ and $5 \AA$, respectively, and $B_{\mathrm{hf}}$ follows a $T^{3 / 2}$ law. For $t_{\mathrm{Fe}}=2 \AA$, spin-glasslike behavior was observed by magnetometry.

Metallic multilayered films offer an exciting field for the exploration of magnetic properties in novel systems and at interfaces. ${ }^{1}$ In a search for new multilayers with interesting magnetic behavior we have studied the Fe-Rh system. According to the thermodynamic $\mathrm{Fe}-\mathrm{Rh}$ phase diagram ${ }^{2}$ a wide solubility range exists at rather low temperatures on both the Fe-rich and Rh-rich side. Therefore, a tendency for interfacealloy formation may be expected in these multilayers. The properties of $\mathrm{Fe} / \mathrm{Rh}$ interfaces are unknown so far.

We have prepared a series of $\mathrm{Rh} / \mathrm{Fe}$ multilayers with constant $\mathrm{Rh}$ thickness $(20 \AA)$ by alternating evaporation of $\mathrm{Rh}$ and ${ }^{57} \mathrm{Fe}$ isotope (95\% enriched) in an UHV system. The pressure during cvaporation was $<5 \times 10^{-9}$ mbar. The substrates were polyimid foils for Mössbauer and magnetometric studies and $\mathrm{Si}$ wafers for small- and large-angle $\mathrm{x}$-ray diffraction (XRD). To reduce intermixing, the substrate temperature was held at $\sim 100 \mathrm{~K}$ during multilayer growth. $\mathrm{Rh}$ and ${ }^{57} \mathrm{Fe}$ were evaporated from a $2-\mathrm{kW}$ electron-beam gun and a small resistively heated evaporation cell with alumina crucible, respectively. Four different types of multilayers have been prepared, namely $\left[\operatorname{Rh}(20 \AA) /{ }^{57} \mathrm{Fe}(2 \AA)\right]_{100}+\operatorname{Rh}(20$ $\AA),\left[\operatorname{Rh}(20 \AA) /{ }^{57} \mathrm{Fe}(5 \AA)\right]_{52}+\operatorname{Rh}(20 \AA),\left[\operatorname{Rh}(20 \AA) /{ }^{57} \mathrm{Fe}(10\right.$ $\AA)]_{30}+\operatorname{Rh}(20 \AA)$, and $[\operatorname{Rh}(20 \AA) / \mathrm{Fe}(15 \AA)]_{20}+\operatorname{Rh}(20 \AA)$. (All samples were coated by $20 \AA \mathrm{Rh}$ for protection.)

The small-angle XRD patterns (Fig. 1) exhibit a clear first-order superstructure Bragg peak for all samples, and an additional second-order peak for $t_{\mathrm{Fe}}=10$ and $15 \AA$. This and the fact that no higher-order superstructure peaks have been detected (not shown in Fig. 1) demonstrates qualitatively that our samples are compositionally modulated structures with some degree of intermixing at the interfaces. The multilayer periodicity determined from Fig. 1 is $23.7,24.9,29.7$, and $37.7 \AA$ for $t_{\mathrm{Fe}}=2,5,10$, and $15 \AA$, respectively, being in agreement with the nominal periodicity within $7 \%$ or better. The large-angle XRD patterns from our samples (not shown) exhibit a dominant fcc $\mathrm{Rh}(111)$-Bragg peak and weaker peaks from higher-indexed $\mathrm{Rh}$ planes. However, the $\mathrm{Rh}$ peaks were found to be shifted slightly to higher Bragg angles upon increase of $t_{\mathrm{Fe}}$, implying a fcc-lattice parameter $\left(a_{0}\right)$ reduction with increasing Fe-film thickness from $a_{0}=3.808 \AA$ (pure $\mathrm{Rh} \mathrm{film}$ ) to $3.793,3.780,3.738$, and $3.713 \AA$ for $t_{\mathrm{Fe}}=2,5,10$, and $15 \AA$, respectively. No pure
bcc-Fe Bragg peaks could be detected. However, a shoulder (near $2 \theta \approx 40^{\circ}$ ) on the low-angle side of the $\operatorname{Rh}(111)$ peak observed for the thicker Fe films (10 and $15 \AA$ ) may be assigned to a bcc (110) reflex with a corresponding beclattice parameter which is enhanced (relative to that of pure bcc $\mathrm{Fe}$ ) by $11.4 \%$ (for $t_{\mathrm{Fe}}=10 \AA$ ) and $10.0 \%$ (for $t_{\mathrm{Fe}}=15 \AA$ ). In view of the small-angle XRD and Mössbauer results (below) the observed reduction or increase in lattice parameter is interpreted by the main effect of interface-fcc-alloy formation or bcc-alloy formation, respectively. For fcc Fe-Rh alloys, our interpretation is qualitatively supported by the known decrease of $a_{0}$ with rising $\mathrm{Fe}$ content in the bulk. ${ }^{3}$

Mössbauer spectra measured at $4.2 \mathrm{~K}$ (Fig. 2) indicate magnetic hyperfine (hf) splitting at all Fe thicknesses. These spectra were least-squares fitted using a histogram distribution, ${ }^{4} P\left(B_{\mathrm{hf}}\right)$, of hyperfine fields $B_{\mathrm{hf}}$. It was found necessary to include a small linear correlation between isomer shift $\delta$ and $B_{\text {hf }}$, given by about $+0.004 \mathrm{~mm} \mathrm{~s}^{-1} / \mathrm{T}$.

For $t_{\mathrm{Fe}}=2 \AA$, the most-probable (peak) hf field, $B_{\mathrm{hf}}^{\text {peak }}$, has a value of $16.8 \mathrm{~T}$ (Fig. 2) which is typical for that of a $\sim 7$ at. \% Fe disordered fcc-Fe-Rh bulk alloy at $4.2 \mathrm{~K}^{5}$ This

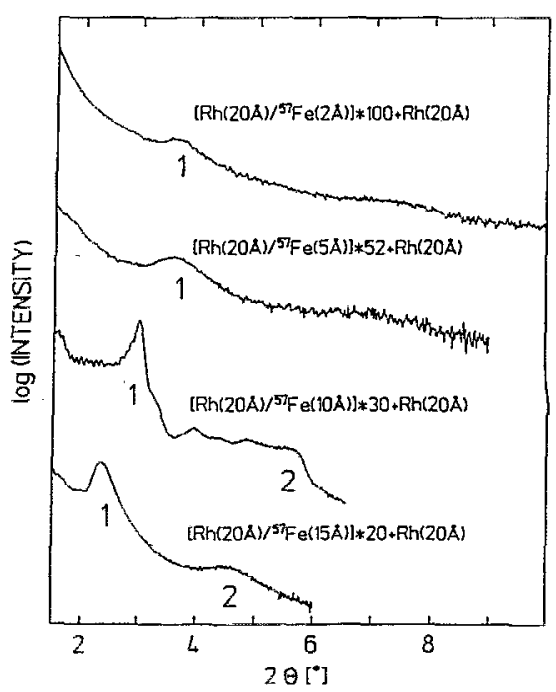

FIG. 1. Small-angle XRD patterns of $\mathrm{Rh}(20 \AA) /^{57} \mathrm{Fe}\left(t_{\mathrm{Fe}}\right)$ multilayers with $t_{\mathrm{Fe}}=2,5,10$, and $15 \AA$ (from top) (Cu $K \alpha$ radiation). 


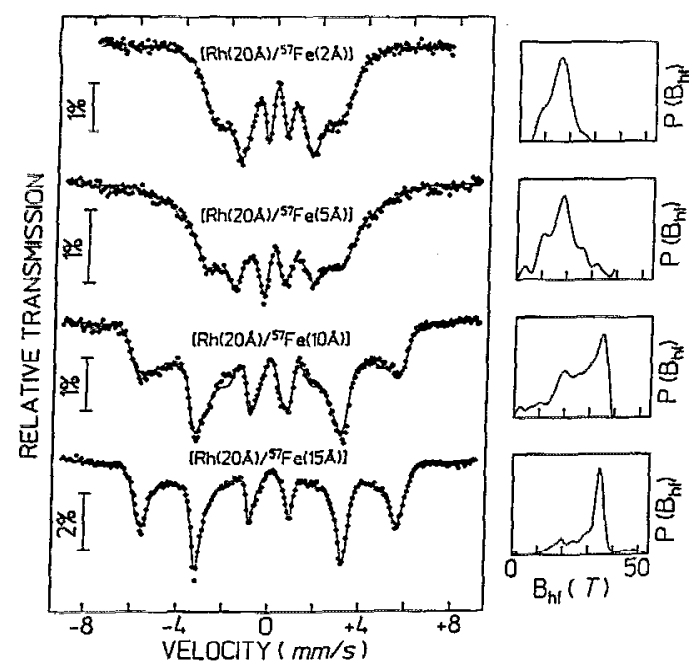

FIG. 2. Mössbauer spectra measured at $4.2 \mathrm{~K}$ and distributions $P\left(B_{\mathrm{hf}}\right)$ of $\mathrm{Rh}(20 \AA) \rho^{57} \mathrm{Fe}\left(t_{\mathrm{Fe}}\right)$ multilayers for $t_{\mathrm{Fe}}=2,5,10$, and $15 \AA$ (from top).

suggests that the $2 \AA^{57} \mathrm{Fe}$ film [ $\sim 1$ monolayer (ML) bcc $\mathrm{Fe}(110)]$ is completely alloyed with $\mathrm{Rh}$ resulting in an fcc$\mathrm{Fe}$-Rh alloy of average $\mathrm{Fe}$ concentration $c_{\mathrm{he}}^{B_{\mathrm{hi}}}$ of $\sim 7$ at. \%. In the case of $t_{\mathrm{Fe}}=5 \AA, P\left(B_{\mathrm{hf}}\right)$ in Fig. 2 shows a pronounced peak at 18.2 $\mathrm{T}$ which demonstrates that this is also a pure fcc-RhFe multilayer. This hf field corresponds to $c_{\mathrm{Fe}}^{B_{\mathrm{hf}}}$ $\sim \overline{19}$ at. $\%$, according to Ref. 5 . We have also estimated the Fe concentration $\left(c_{\mathrm{Fe}}^{a_{0}}\right)$ in the fcc-alloy layer by comparing the measured lattice parameters $\left(a_{0}\right)$ with those of bulk alloys. ${ }^{3}$ Table I shows that $c_{\mathrm{Fe}}^{a_{0}}$ values for 2 and $5 \AA \mathrm{Fe}$ are in rough agreement with corresponding $c_{\mathrm{Fe}}^{B_{\mathrm{hf}}}$ values. The calculated $\mathrm{Rh}$ thicknesses, $t_{\mathrm{Rh}}^{\mathrm{fcc} \text { alloy }}$, required for explaining the obtained $c_{\mathrm{Fe}}^{B_{\mathrm{he}}}$ or $c_{\mathrm{Fe}}^{a_{0}}$ values are given in Table $\mathrm{I}$, too. (It was assumed that $t_{\mathrm{Fe}}=2$ and $5 \AA$ are completely alloyed). The $t_{\mathrm{Rh}}^{\text {foc alloy }}$ values obtained from $c_{\mathrm{Fe}}^{B_{\mathrm{hf}}}$ appear somewhat too large as compared to the deposited $20 \AA$ - $\mathrm{Rh}$ layer, while those values deduced from $c_{\mathrm{Fe}}^{a_{0}}$ appear to be reasonable. Table I indicates also the $\mathrm{Fe}$ concentration $\left(c_{\mathrm{Fe}}^{T_{f}}\right)$ obtained by com-

TABLE I. Estimated Fe concentrations $c_{\mathrm{Fe}}^{T_{f}}, c_{\mathrm{Fe}}^{B_{\mathrm{hf}}}$ and $c_{\mathrm{Fe}}^{a_{0}}$ of the fcc and bcc $\mathrm{Rh}-\mathrm{Fe}$ alloy phase in $\mathrm{Rh} / \mathrm{Fe}$ multilayers. $t_{\mathrm{Rh}}^{\mathrm{fcc} \text { alloy }}$ is the calculated $\mathrm{Rh}$ thickness consumed in fcc-alloy formation.

\begin{tabular}{|c|c|c|c|c|}
\hline $\mathrm{Rh}(20 \AA) / \mathrm{Fe}\left(t_{\mathrm{Fe}}\right)$ & $t_{\mathrm{Fe}}=2 \AA$ & $t_{\mathrm{Fe}}=5 \AA$ & $t_{\mathrm{Fe}}=10 \AA$ & $t_{\mathrm{Fe}}=15 \AA$ \\
\hline \multicolumn{5}{|l|}{ fcc phase } \\
\hline$c_{\mathrm{Fe}}^{T_{f}} /$ at. \% & 7 & 12 & 24 & $\cdots$ \\
\hline$\left(t_{\mathrm{Rh}}^{f \infty}\right.$ alloy $)$ & (25 ̊̊?) & (34 Å?) & (9.4 ̊̊?) & $\cdots$ \\
\hline$c_{\mathrm{Fe}}^{B_{\mathrm{bf}}} / \mathrm{at} . \mathscr{\mathscr { C }}$ & $\sim 7$ & $\sim 19$ & $>25$ & $>25$ \\
\hline$\left(t_{\mathrm{Rh}}^{\mathrm{foc}}\right.$ alloy $)$ & (25 Å?) & (20 Å?) & $\cdots$ & $\cdots$ \\
\hline $\begin{array}{l}c_{\mathrm{Fe}}^{a_{0}} / \text { at. \% } \\
\left(t_{\mathrm{Rh}}^{\mathrm{foc}} \text { alloy }\right)\end{array}$ & $\begin{array}{c}10 \\
(17 \dot{A})\end{array}$ & $\begin{array}{c}24 \\
(14.8 \AA)\end{array}$ & $\begin{array}{c}50 \\
(3.0 \AA)\end{array}$ & $\begin{array}{c}62 \\
(1.2 \AA)\end{array}$ \\
\hline \multicolumn{5}{|l|}{ boc phase } \\
\hline$c_{\mathrm{Fe}}^{B_{\mathrm{ht}} / \mathrm{at} .9 \%}$ & $\cdots$ & $\cdots$ & -96 & $\sim 96$ \\
\hline
\end{tabular}

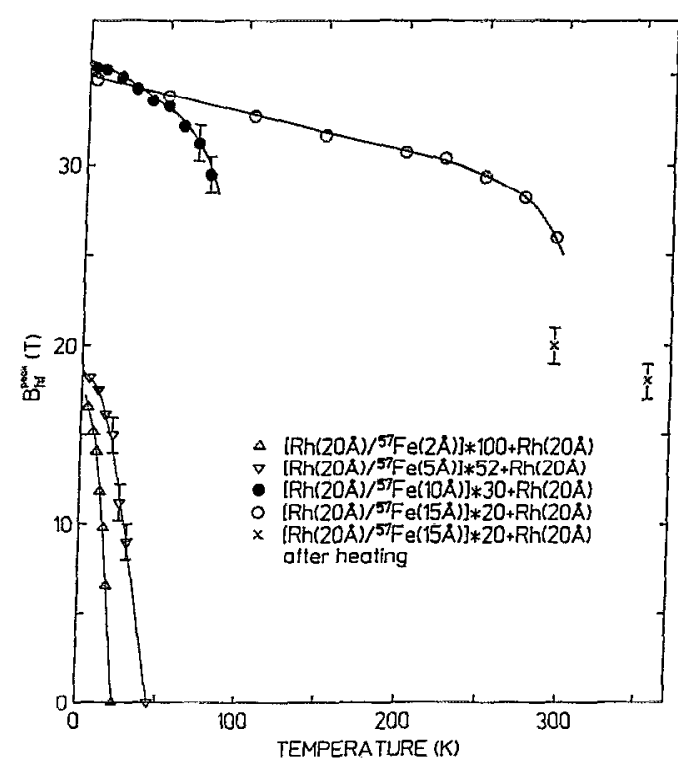

FIG. 3. Temperature dependence of $B$ peak for $t_{\mathrm{re}}=2 \AA(\Delta), 5 \AA(\nabla), 10 \AA$ (Ө) and $15 \AA(O)$. Crosses $(X)$ : for $t_{\mathrm{Fe}}=15 \AA$ after annealing at $355 \mathrm{~K}$.

paring the measured (average) magnetic transition temperature $T_{f}$ of our films $\left(T_{f}=23 \pm 4 \mathrm{~K}\right.$ for $t_{\mathrm{Fe}}=2 \AA$ and $T_{f}=45 \pm 7 \mathrm{~K}$ for $t_{\mathrm{Fe}}=5 \AA$, respectively, see Fig. 3) with the $T_{f}$ vs $c_{\mathrm{Fe}}$ behavior of disordered fcc-RhFe alloys; 5 however, the corresponding (calculated) $t_{\mathrm{Rh}}^{\mathrm{fcc} \text { alloy }}$ values (Table I) are unreasonably high (e.g., $34 \AA$ for $t_{\mathrm{Fe}}=5 \AA$ ). This very likely indicates that the $T_{f}$ vs $c_{\mathrm{Fe}}$ behavior in ultrathin $\mathrm{RhFe}$ alloy layers deviates from that in bulk alloys as is the case for other spin glasses. ${ }^{6}$ From the Mösbauer-lineintensity ratios the Fe-spin orientation in these samples was found to be nearly random which can be expected for spinglass-type magnetism where a magnetic shape anisotropy is absent.

One can notice in Fig. 2 that the $P\left(B_{\mathrm{hf}}\right)$ curves (and the spectra) change drastically with increasing Fe thickness: the fcc-alloy distribution peak near $\sim 17 \mathrm{~T}$ decreases gradually in relative intensity and shifts to slightly higher values, while simultancously a new peak ncar $36 \mathrm{~T}(35 \mathrm{~T})$ evolves and dominates at $t_{\mathrm{Fe}}=10 \AA$ (or $t_{\mathrm{Fe}}=15 \AA$ ). This new peak is assigned to a disordered ferromagnetic bcc-Fe-Rh alloy. This follows from a comparison of our $B_{\mathrm{hf}}^{\text {peak }}$ values with those of Fe-rich disordered bcc-Fe-Rh bulk alloys. ${ }^{7}$ We may estimate the average composition $c_{\mathrm{Fe}}$ of the bcc alloy in our multilayers by using the $B_{\mathrm{hf}}$ vs $c_{\mathrm{Fe}}$ curve in Ref. 7 (with $B_{\mathrm{hf}}$ in Ref. 7 corrected by a factor of 1.03 for the low temperature (4.2 $\mathrm{K})$ used in our case). This leads to $c_{\mathrm{Fe}} \sim 96$ at. $\%$ for $t_{\mathrm{Fe}}=10$ and $15 \AA$ in the bcc alloy (Table I).

The shape of the distribution $P\left(B_{\mathrm{hf}}\right)$ for $t_{\mathrm{Fe}}=10 \AA$ (Fig. 2) demonstrates that fcc and bcc alloy phases coexist in this multilayer, (with the fcc-alloy distribution peak located at $19.8 \mathrm{~T}$ ). Even for $t_{\mathrm{Fe}}=15 \AA$, the fcc-alloy distribution peak (at $20.0 \mathrm{~T}$ ) is still present, together with a low-field distribution part (for $B_{\mathrm{hf}}<28 \mathrm{~T}$ ). The relative area of the low-field or high-field $\left(B_{\text {hf }}>28 \mathrm{~T}\right)$ distribution part provides values for the relative phase content (fcc or bcc) in the multilayer. In the case of $t_{\mathrm{Fe}}=10 \AA$ we find that $(36 \pm 15) \%$ of the Fe atoms form the fcc-interface alloy (and 64\% the bcc phase); for 


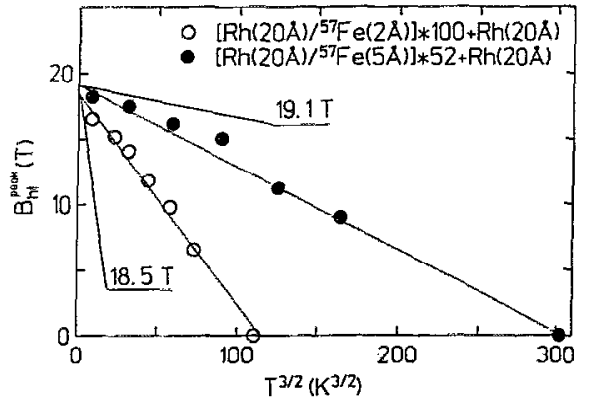

FIG. 4. $B_{\mathrm{hf}}^{\text {peak }}$ vs $T^{3 / 2}$ for $t_{\mathrm{Fe}}=2 \AA(O)$ and $5 \AA(\bullet)$.

$t_{\mathrm{Fe}}=15 \AA$, the corresponding values are $(30 \pm 5) \%$ (fcc) and $70 \%$ (bcc). This means that in the average a thickness of 3.6 $\AA \mathrm{Fe}$ of the original $10 \AA \mathrm{bcc}-\mathrm{Fe}$ layer is transformed to the fcc-interface alloy by interdiffusion; for the $15 \AA \mathrm{Fe}$ film, the corresponding value is $4.5 \AA \mathrm{Fe}$. The Fe-spin direction of the bcc phase was observed to be preferentially oriented in the film plane indicating a shape anisotropy due to ferromagnetism.

It follows from the temperature dependence of $B$ pf (Fig. 3) that the magnetic transition temperatures of the bcc phase (in multilayers with $t_{\mathrm{Fe}}=10$ and $15 \AA$ ) are much higher than those of the fcc phase (with $t_{\mathrm{Fe}}=2$ and $5 \AA$ ). For $t_{\mathrm{Fe}}=2$ and $5 \AA, B_{\mathrm{hf}}^{\text {peak }}(T)$ follows closely a $T^{3 / 2}$-spin wave law over the whole temperature range (Fig. 4). For $t_{\mathrm{Fe}}=15 \AA, B_{\mathrm{hf}}^{\text {peak }}$ shows a remarkable linear $T$ dependence over a wide $T$ range. It has been suggested that such a behavior is related to superparamagnetic relaxation of bcc-phase clusters. ${ }^{8}$ However, as we have not observed a remarkable change at $150 \mathrm{~K}$ in the central component of the spectrum (not shown) even by applying fields up to $1 \mathrm{~T}$, we may exclude superparamagnetism. Therefore, the linear $\mathrm{T}$ dependence observed can be explained by quasi-two-dimensional behavior of the bcc phase in the $15-\AA$ sample, as predicted theoretically. ${ }^{9}$ In contrast, the $t_{\mathrm{Fe}}=10 \AA$ multilayer has been observed to be superparamagnetic by applying a magnetic field. After a measurement at $355 \mathrm{~K}$ and recooling to $295 \mathrm{~K}$, an irreversible drop of $B_{\mathrm{hf}}^{\text {peak }}$ at $295 \mathrm{~K}$ occured. As we did not observe a change in $P\left(B_{\mathrm{hf}}\right)$ at $4.2 \mathrm{~K}$ after annealing, this drop in $B$ hf

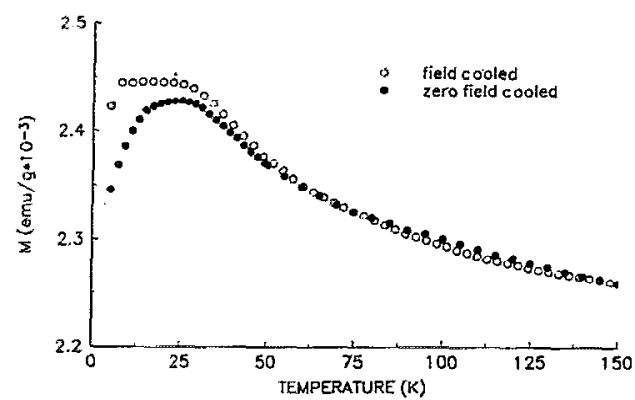

FIG. 5. Temperature dependence of magnetization in a $\mathrm{Rh}(20 \AA) /^{57}$ $\mathrm{Fe}(2 \AA)$ multilayer measured in $B_{\text {ext }}=0.5 \mathrm{~T}$, zero-field cooled $(\Theta)$ and field cooled (O).

could be due to a change in bcc Fe-film morphology (island formation) and a resulting change in the $T$ dependence of $B_{\text {hf }}^{\text {peak }}$ (possibly due to superparamagnetism).

The temperature dependence of the magnetization in the multilayer with $t_{\mathrm{Fe}}=2 \AA$ (Fig. 5) indicates typical spinglasslike behavior, i.e., different branches after zero-field cooling and during field cooling. It can be seen that the temperature of the maximum in the zero-field cooled magnetization $(23 \pm 2 \mathrm{~K})$ is in very good agreement with the magnetic transition temperature obtained from Mössbauer spectroscopy. This proves that interface fcc alloys with spin-glasslike properties can be obtained in $\mathrm{Rh} / \mathrm{Fe}$ multilayers.

We are grateful to $U$, von Hörsten for technical assistance and sample preparation. This work was supported by the DFG (SFB166).

${ }^{1}$ For an overvicw of recent work, see Proccedings of the First Intcrnational Symposium on Metallic Multilayers, Kyoto, Japan, 1993 [J. Magn. Magn. Mater 126 (1993)].

${ }^{2}$ O. Kubaschewski, Iron Binary Phase Diagrams (Springer, Berlin, 1992).

${ }^{3}$ C. C. Chao, P. Duwez, and C. C. Tsuei, J. Appl. Phys. 42, 4282 (1971).

${ }^{4}$ J. Hesse and A. Rübartsch, J. Phys. E 7, 526 (1974).

${ }^{5}$ B. Window, G. Longworth, and C. E. Johnson, J. Phys. C 3, 2156 (1970).

${ }^{6} \mathrm{H}$. Vloeberghs et al., Europhys. Lett. 12, 557 (1990).

${ }^{7}$ G. Shirane, C. W. Chen, P. A. Flinn, and R. Nathans, J. Appl. Phys. 34, 1044 (1963).

${ }^{8}$ Z. Q. Qiu, S. H. Mayer, C. J. Gutierrez, H. Tang, and J. C. Walker, Phys. Rev. Lett. 63, 1649 (1989).

${ }^{9}$ J. C. Levy and J. L. Motchane, J. Vac. Sci. Technol. 9, 721 (1971); J. A. Davis, J. Appl. Phys. 36, 3520 (1965). 